\title{
Special Issue on Soil Pollution, Control, and Remediation
}

\author{
Yan $\mathrm{He}^{1,2}$, Peng Cai ${ }^{3}$ \\ 1 Zhejiang Provincial Key Laboratory of Agricultural Resources and Environment, College of Environmental and Resource Sciences, \\ Zhejiang University, Hangzhou 310058, China \\ 2 Key Laboratory of Environment Remediation and Ecological Health, Ministry of Education, Hangzhou 310058, China \\ 3 State Key Laboratory of Agricultural Microbiology, College of Resources and Environment, Huazhong Agricultural University, \\ Wuhan 430070, China
}

(c) Higher Education Press 2021

In the past decades, toxic pollutants have increasingly accumulated in the soil system due to intensifying agricultural, industrial, and commercial activities. Soil pollution is a growing global concern, posing significant threats to food security, ecological sustainability, and human health. Research on soil pollution, control, and remediation is becoming a frontier in resource and environmental sciences, and however, also a challenge for scientists. Soil is usually a sink of many kinds of toxic inorganic, organic and biological pollutants, including heavy metals, pesticides, antibiotics, phthalic esters, environmental hormones, antibiotics resistance genes, pathogens, etc. We are facing the great challenges in protecting our environment from contamination by these toxic pollutants.

To sustain soil environment, impressive progress has been made over the last years focusing on areas ranging from agricultural chemistry, soil-water resource management and soil health to environmental science \& technology and pollution control \& remediation technology. A platform to present the state-of-the-art research findings is necessary for scientists and professionals working on toxic pollutants across multiple disciplines. We invite Prof. Yetao Tang (Sun Yat-sen University, China), Prof. Linchuan Fang (Northwest A\&F University, China) and Dr. Songcan Chen (Helmholtz Centre for Environmental Research - UFZ, Germany) as co-guest editors to jointly call for papers in this aspect to discuss how the knowledge can be used for protecting our soil. In this special issue, a few of the many aspects regarding the biogeochemistry of inorganic, organic and biological pollutants for improved soil ecosystem services and human health are discussed. Topics such as interactions between soil components and toxic pollutants and their impact on soil ecology, biogeochemistry and processes of toxic pollutants in soil ecosystem, pollutants dynamics and fate in the soil-plant system, novel techniques in soil micro-ecological environment and new approaches in ecological control and remediation of contaminated soil are covered.

In the future, a substantial advance is foreseeable in our understanding of soil pollution ecology that supports an improved solution to soil pollution control and remediation, and thereby polluted soil health reconstruction. It will be exciting to probe how the biogeochemical processes of toxic pollutants are regulated by the food web of below-ground soil organisms and their cascading effects. Researches tackling this cutting-edge issue will lead to many innovations in soil pollution control and remediation that address China's great strategic needs. More progress will be achieved based on an interdisciplinary work in the natural sciences, agricultural sciences, life sciences and medicine.

\section{Guest Editors of the Special Issue:}

Prof. Yan $\mathrm{He}$

College of Environmental \& Resource Sciences,

Zhejiang University, China

yhe2006@zju.edu.cn

Prof. Peng Cai

College of Resources and Environment,

Huazhong Agricultural University, China

cp@mail.hzau.edu.cn

Prof. Yetao Tang

School of Environmental Science and Engineering,

Sun Yat-sen University, China

eestyt@mail.sysu.edu.cn

Prof. Linchuan Fang

State Key Laboratory of Soil Erosion and Dryland Farming on the Loess Plateau, Northwest A\&F

University, China

flinc629@hotmail.com 
Dr. Songcan Chen

Department of Isotope Biogeochemistry, Helmholtz

Centre for Environmental Research - UFZ, Germany

songcan.chen@ufz.de 